\title{
»l'm down on my knees«: Körper im Gebet
}

\section{Ein Essay}

\author{
Natalie Fritz / Anna-Katharina Höpflinger
}

"Life is a mystery

Everyone must stand alone I hear you call my name And it feels like home. ${ }^{1}$

Mit diesen Zeilen aus dem Lied "Like a Prayer" verweist die USamerikanische "Queen of Pop«, Madonna (eine der erfolgreichsten populären Musikerinnen unserer Zeit) auf die Orientierungslosigkeit in einer zunehmend unübersichtlichen Welt und liefert auch gleich eine Lösung: Das Gebet als Zeichen einer körperlichen und geistigen Hinwendung zu einer Gottheit vermittelt laut Madonna Geborgenheit und Sicherheit.

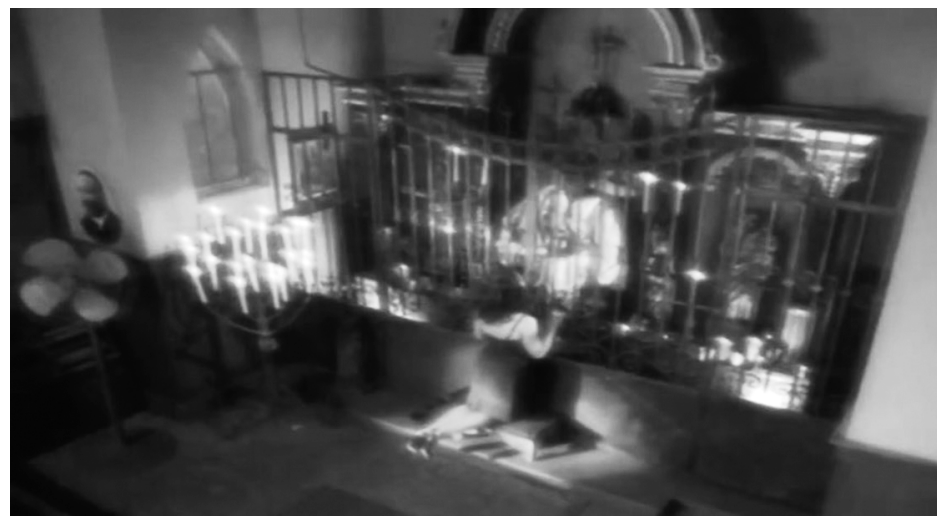

Abbildung 1: Madonna kniet vor einem schwarzen Heiligen

(Like a Prayer, 00:01:14)

Ist die Betonung des Körperlichen beim Beten lediglich eine von Madonnas ausgeklügelten Marketingstrategien? Der folgende Text möchte zeigen, dass dem nicht so ist. Beim Gebet handelt es

\footnotetext{
I Madonna, Like a Prayer, 1989, Sire; Warner Bros., Johnny Yuma Studios (Burbank, California).
} 
sich stets um eine mehr oder minder ritualisierte Inszenierung der Zwiesprache zwischen einem oder mehreren Individuen und einer (oder mehreren) Gottheit(en), zu der das Körperliche wesentlich dazugehört.

An der letzten Fussballweltmeisterschaft in Brasilien wurde diese performative Qualität des Gebets besonders deutlich vor Augen geführt: Beten ist nicht nur die Praxis einiger besonders Frommer im "stillen Kämmerlein" oder in religiösen Gebäuden - auch heute kann es zur öffentlichen Handlung werden. So schwenkte an der Fussball-WM während besonders kritischen Szenen die Kamera gerne auf die Zuschauertribüne und übermittelte dem Publikum zuhause vor dem Fernseher die nervenaufreibende Atmosphäre im Stadion. Man sah, wie sich die Anspannung und die Hoffnung auf einen Sieg der eigenen Mannschaft innerhalb der Fanmasse in ganz unterschiedlicher Form äussern kann: Die einen bissen die Zähne zusammen und drückten die Daumen, die anderen schlossen die Augen im verzweifelten Versuch, einen schlechten Ausgang ungesehen zu machen; wieder andere hielten sich in den Armen. Überraschenderweise fanden sich aber auch eine beachtliche Anzahl an Kameraeinstellungen, die betende Menschen zeigten: Die Fans beteten allein oder in der Gruppe - und zwar smit vollem Körpereinsatz - für einen Sieg ihrer Mannschaft (Abb. 2).

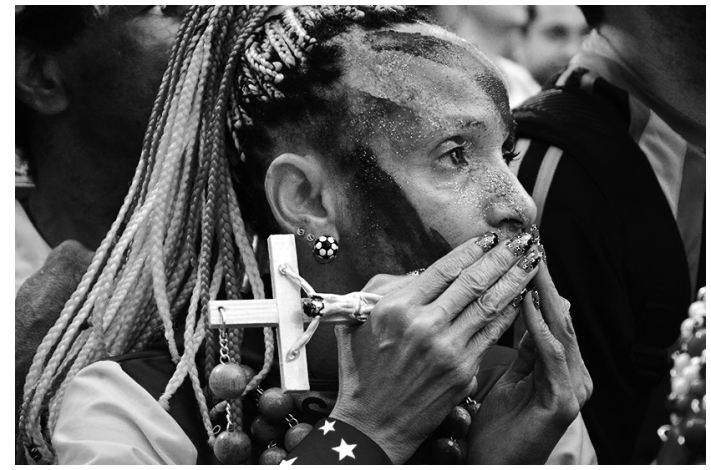

Abbildung 2: Eine Brasilienanhängerin hofft und betet für einen Sieg ihrer Mannschaft im Viertelfinale gegen Kolumbien (C) Ben Taverner, https://www.flickr.com/photos/bentavener/ sets/72157645519328755).

Das Gebet dient im sportlichen Kontext einem spezifischen Ziel und verbindet individuellen Glauben mit einem kollektiven Wunsch. 
Im Fussballstadion wird es zum Zeichen von sichtbarem Mitgefühl und Miterleben, von Identifikationsprozessen, einer spezifischen Verhaltensstrategie und einer modernen Verehrungspraxis. Die Fans beten für sich und ihre Helden, die für die Ehre des Heimatlandes das Runde ins Eckige zu versenken versuchen. Ein Motiv dafür ist, dass das mit einem Sieg assoziierte Gefühl der Zugehörigkeit (einer fiktiven nationalen Einheit, wie es sie in der sozialen Realität so vielleicht nicht gibt) überwältigend ist und für den kurzen $\mathrm{Au}-$ genblick des Freudentaumels alle gleich macht. Der viel diskutierte "zwölfte Mann", das Stadionpublikum, ist mit seinen Gesten der Unterstützung Ansporn für die Mannschaft. So wird auf der Ebene der Zuschauer und Zuschauerinnen das körperlich inszenierte Gebet -, das von utilitaristischem Charakter ist, zu einem Signal stilisiert, das sowohl die menschliche als auch die göttliche Unterstützung symbolisiert.

Auch die Fussballspieler selbst versuchen, den Zuschauern und Zuschauerinnen in der Arena und am Bildschirm anhand eindeutiger Gesten zu vermitteln, wie ernst sie den spielerischen Kampf um den Ball nehmen. Dabei tritt auch die Religion auf den Plan: Immer häufiger sieht man, wie verschiedene Mannschaften vor dem Anpfiff vor Tausenden von Zuschauern und Zuschauerinnen auf dem Spielfeld beten oder nach einem bedeutenden Tor für ein gemeinsames Gebet zusammenkommen. Ein solches Gebet kann auf der Ebene der Spieler mit verschiedenen Funktionen verkettet werden: Es dient der Beruhigung der Nerven, fördert den sozialen Zusammenhalt der Mannschaft, repräsentiert die Identität eines Landes und kann das "Unkontrollierbare", also den Spielverlauf, in die Kontrolle zu überführen suchen. Dabei kann dieser letzte Punkt auch mit einem do ut des-Gedanken verbunden sein: Die Gottheit verleiht den Sieg denen, die sich um sie kümmern. Dahinter steht ein Gedankengut, das bereits in der Antike zu beobachten ist: Sportliche Wettkämpfe waren damals religiöse Rituale; die Sieger wurden von den Gottheiten auserwählt.

Das Beispiel der Fussball-WM zeigt, dass Beten eine vielschichtige, den Körper einschliessende Handlung darstellt, die auch heute in verschiedensten Regionen des menschlichen Lebens eine Rolle spielen kann, sich also nicht auf im engeren Sinne religiöse Bereiche beschränken lässt. Im Folgenden werden wir den Fokus auf beobachtbare Dimensionen des Gebets legen: Körperhaltungen, Inszenierungen und Repräsentationen. Wir konzentrieren uns also auf das Gebet als Handlung und Praxis. 


\section{Der Körper als Medium}

» hear your voice It's like an angel sighing I have no choice, I hear your voice Feels like flying«

Das Gebet stellt keine monologische Situation dar, sondern - wie Madonna wunderbar bildhaft umschreibt -, einen dialogischen Akt. Madonna verweist in den obigen Zeilen auf das physische und das religiös-emotionale Sprach- und Hörvermögen als wichtige Voraussetzung für eine fruchtbare Auseinandersetzung mit dem Transzendenten. Um Wünsche und Nöte ausdrücken und Ratschläge empfangen zu können, greift der Mensch ganz selbstverständlich auf seinen Körper zurück: er fleht mit lauter Stimme, kniet mit ehrfürchtig gesenktem Kopf, tanzt ekstatisch zu einem Gospel ...
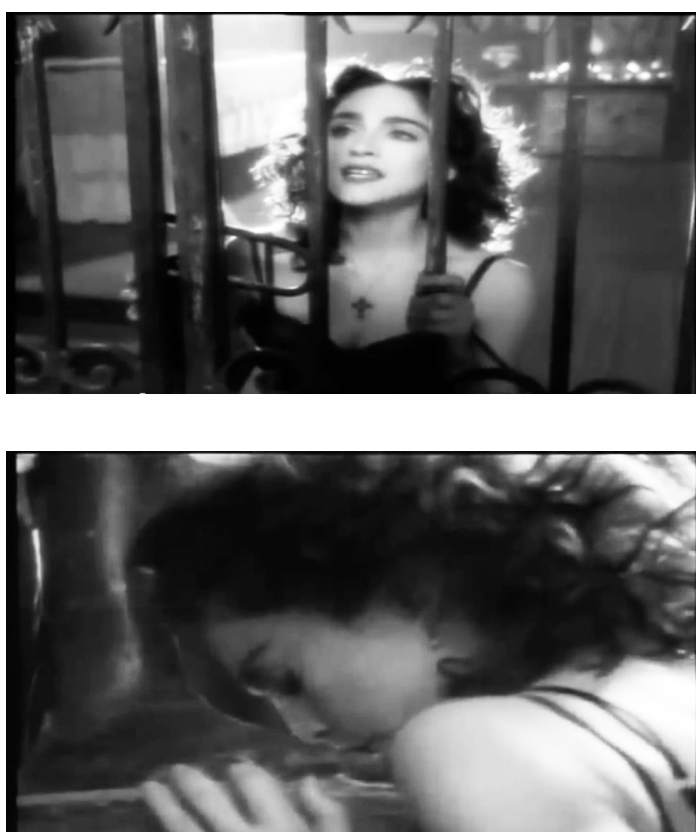

Abbildung 3 a, b: Madonna hält intensive Zwiesprache mit dem schwarzen Heiligen um ihm kurz darauf in einem Akt der Demut die Füsse zu küssen (Like a Prayer, 00:01:10; 00.01:34). 
Im Gebet wird der Körper zum hauptsächlichen Kommunikationsmedium. Es sind in erster Linie die spezifischen Gesten, die Lautstärke der Stimme und die Bekleidung, die zusammen mit einer bestimmten räumlichen und zeitlichen Strukturierung ein Gebet ausmachen und von anderen Kommunikationsformen abgrenzen. Die Gestik und die Inszenierung machen ein Gebet auch von aussen als ein solches erkennbar. Gesten sind kulturspezifisch und werden durch Sozialisation (oder eine andere Form der Aneignung) erlernt. Adressaten und Adressatinnen des Gebetes sind zunächst transzendente Mächte. Entsprechend ist ein wichtiger Aspekt der Gebetsvorbereitung, den Körper von seiner besten Seite zu zeigen: Sauber muss der Körper sein, der sich an eine transzendente Macht wendet. Die rituelle Reinigung vom alltäglichen Schmutz fungiert demnach auch als Phase der Vorbereitung für den Dialog mit der Gottheit.

Ein Gebet kann folglich als mehr oder minder regulierter Ablauf verschiedener "Hinwendungs-Phasen" verstanden werden, wobei der eigentliche Akt der Zwiesprache lediglich einen Teil dieses Geschehens ausmacht. Nicht nur der gesungene, gedachte oder gesprochene Inhalt des Gebetes, sondern auch Gebetsgesten und -haltungen drücken eine bestimmte Beziehung zum Transzendenten aus. Wagt man einen kulturübergreifenden Blick, zeigt sich, dass viele Gebetshaltungen entweder eine Ehrbezeugung oder eine Intimität mit einer transzendenten Macht andeuten. Im Alten Orient gab es beispielsweise die Praxis, sich vor einer Götterstatue (wie vor einem Herrscher) auf den Boden zu werfen, was eine Unterwerfung unter den Willen der Gottheit suggeriert und den Menschen körperlich zu einem Bittsteller macht. Das Senken des Kopfes oder das Niederknien vor einer Statue kann ebenfalls in diese Richtung gedeutet werden. So finden sich auch im Alten Testament Passagen, in denen sich Personen verneigen, zum Gebet niederknien oder niederwerfen (z.B. Gen 24,26). Es sind Gesten, die den Respekt vor Höheren ausdrücken und oft den politischen Ehrbezeugungen, also dem Verhalten vor Königen oder Herrscherinnen, entnommen sind (vgl. bspw. Abb. 3b, 4b und c). 

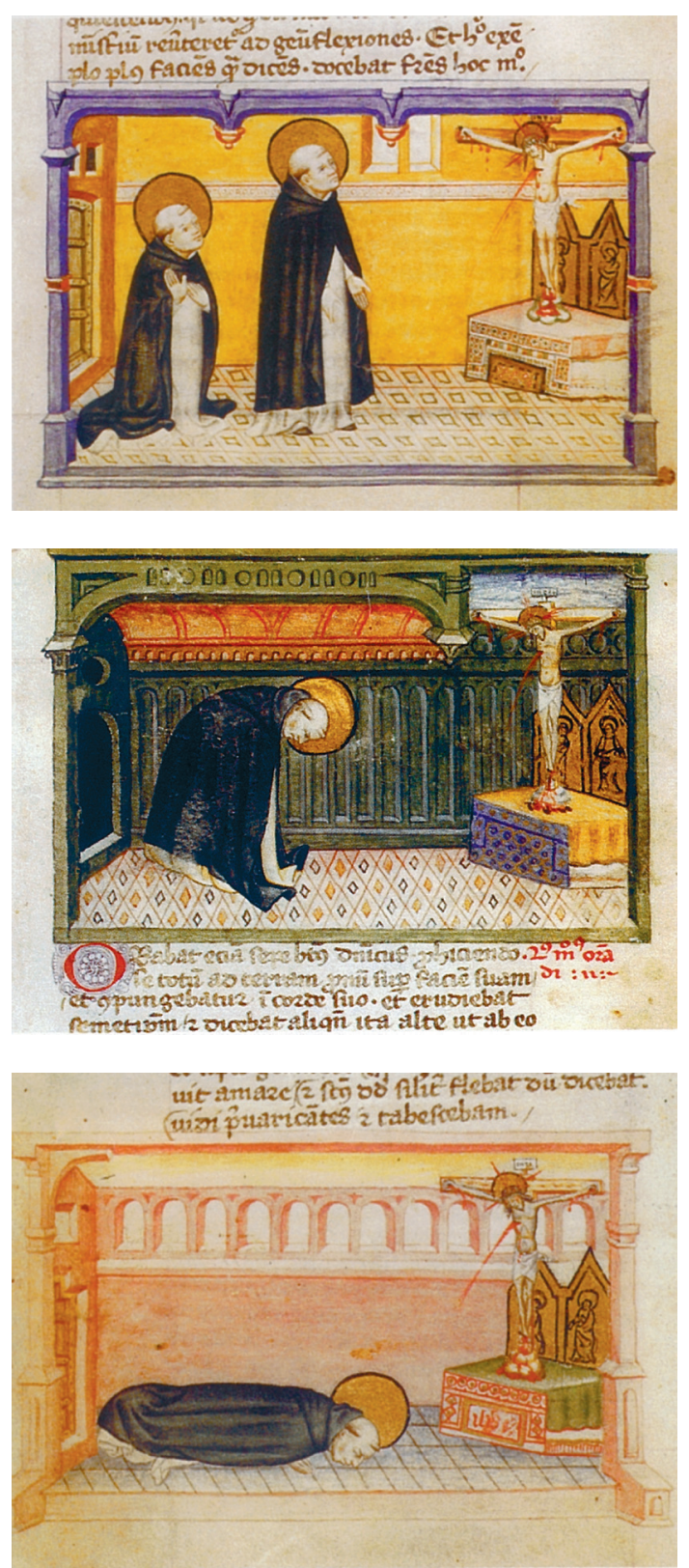

Abbildung $4 \mathrm{a}, \mathrm{b}, \mathrm{c}$ :

Drei Gebetshaltungen nach Dominikus: Knien und Stehen vor Gott, tiefe Verneigung, ausgestrecktes Liegen auf der Erde: http://www. domenicani.net. 
Die stehende Gebetshaltung mit gegen den Himmel erhobenen Armen ist eine ebenfalls gängige Art der körperlichen Inszenierung einer Zwiesprache mit dem Transzendenten (z.B. 1. Kön 8,22f). Das stehende Gebet ist beispielsweise im heutigen jüdischen Kontext vorherrschend; das jüdische "Achtzehngebet" wird deshalb amida, "stehend ", genannt. Das Stehen findet sich auch früh in christlichen Traditionen. So zeigen zum Beispiel Fresken in der Calixtus-Katakombe in Rom die Betenden stehend mit erhobenen ausgestreckten Armen. Das aktive Stehen vor Gott wird dabei zum Symbol für die Gnade, die dem Gläubigen als Nachfolger Christi zuteil wird. Dieser Gestus hat sich heute v.a. in der christlichen Liturgie erhalten, innerhalb deren z.B. römisch-katholische Priester oder lutherische Pfarrer im Sinne eines "Vorbeters" diese Haltung einnehmen können. Das stehende, kollektive Gebet ist in evangelisch-reformierten Gottesdiensten und heute immer häufiger auch in katholischen Messen Usus.

Auf eine intime Zwiesprache mit einer Gottheit deuten dagegen Gesten hin, die zum Beispiel eine Verbindung mit Grusshandlungen aufweisen (Abb. 4a). Ausgestreckte oder erhobene Hände, bei denen die Handflächen gezeigt werden, repräsentieren friedliche Absichten durch ein Unbewaffnetsein. Das lateinische adorare wird mit anbeten übersetzt. Wörtlich bedeutet es jedoch (die Hand) an den Mund führen, bzw. eine Kusshand zuwerfen. Auch dies ist eine Geste der Intimitätsbezeugung; ebenso das Berühren, Streicheln oder Küssen von heiligen Gegenständen während eines Gebets (Abb. 3b).

Ein Gebet kann sowohl einen individuellen als auch einen kollektiven Charakter aufweisen. Die intime Zwiesprache mit einer Gottheit »im stillen Kämmerlein" folgt anderen Inszenierungsstrategien als dies beim gemeinschaftlichen Gebet der Fall ist: Die genau orchestrierten Handlungsabläufe müssen bei Letzterem unbedingt eingehalten werden, da dadurch die Wirkung auf das Kollektiv der Betenden, die sich als Teil eines Ganzen fühlen, verstärkt wird. Hierbei kommen Gesten, die anzeigen, dass der Akt des Betens initiiert oder abgeschlossen wird, eine grosse Relevanz zu. Auch die Lautstärke eines Gebets kann variieren und anzeigen, ob es der Zeitpunkt für eine gemeinschaftliche oder individuelle Kommunikation mit der transzendenten Macht ist. Damit der Aspekt der persönlichen Kommunikation mit der Gottheit auch im kollektiven Gebet nicht zu kurz kommt, sind die körperlichen Abläufe häufig gleichförmig, was einerseits der Konzentration dient, andererseits das Gefühl des Getragenseins verstärkt. So stellt beispielsweise das Beten auf dem 
Kreuzweg im römischen Katholizismus eine mögliche Art der individuellen Kommunikation mit Gott innerhalb eines Kollektivs dar.

Ein Gebet ist jedoch nicht nur eine Kommunikation mit einer transzendenten Macht, sondern vor allem über die Körperhaltung auch eine Botschaft an andere Menschen: Eine betende Person signalisiert ihre Handlung nach aussen, wobei spezifische Reaktionen bei den Beobachtenden ausgelöst werden. Das Eingangsbeispiel der Fussball-Weltmeisterschaft hat diese Funktion des Gebets bereits thematisiert: Es wird emotional reagiert und vielleicht sogar mitgebetet. Eine andere, in der Schweiz oft üblichere Reaktion ist, dass man sich höflich und leise zurückzieht, um die betende Person nicht zu stören.

Das wichtigste Medium sowohl beim stillen Gebet als auch bei einem öffentlichen Gebetsauftritt ist und bleibt also der Körper. Er ermöglicht es dem Individuum, sich einer Gottheit zu nähern und der Aussenwelt zu signalisieren, was es tut.

\section{Der religiöse Raum}

» close my eyes

Oh God I think I'm falling Out of the sky, I close my eyes Heaven help me"

Das Gefühl der Ohnmacht angesichts einer schwierigen Situation, das plötzliche Schweben im luftleeren Raum kann, wie Madonna es besingt, Anlass zu einem Stossgebet sein. Ihr eindringlicher Ruf nach Hilfe hallt durch den Raum und mit geschlossenen Augen erkennt sie, dass sie durch ihren Glauben aufgefangen wird. Der Kirchenraum und die afro-amerikanische christliche Gemeinde werden im Musikvideo zu Madonnas Zufluchtsort.

Verschiedene Dimensionen des Raums in Bezug auf die Betpraxis werden in "Like a Prayer explizit angesprochen: Der Körper ist immer ein Körper im Raum; durch spezifische Körperpraktiken werden also bestimmte Räume geschaffen. Im Gebet können dies sehr unterschiedliche Arten von Räumlichkeiten sein. Einerseits wird, wie bereits angesprochen, eine Art intimer Raum mit einem Ansprechpartner oder einer Ansprechpartnerin geformt. Viele Gebete richten sich zunächst an einen Adressaten oder eine Adressatin, bzw. an ein Kollektivsingular. Dieser intime Raum wird wie oben 
gezeigt auch durch eine bestimmte Körperhaltung kreiert. Sowohl Ehrbezeugungsgesten als auch Gesten der Nähe drücken die Bereitschaft aus, sich auf ein Gegenüber einzulassen.

Gleichzeitig kann im und vor allem durch das Gebet auch ein religiöser Raum erzeugt werden. In Abbildung 5 beispielsweise ist es nur die Körperhaltung des Abgebildeten, die das trostlose Kellerabteil zu einem Raum des Gebets werden lässt und das Bild mit spezifischen Semantiken zu füllen vermag (Abb. 5).

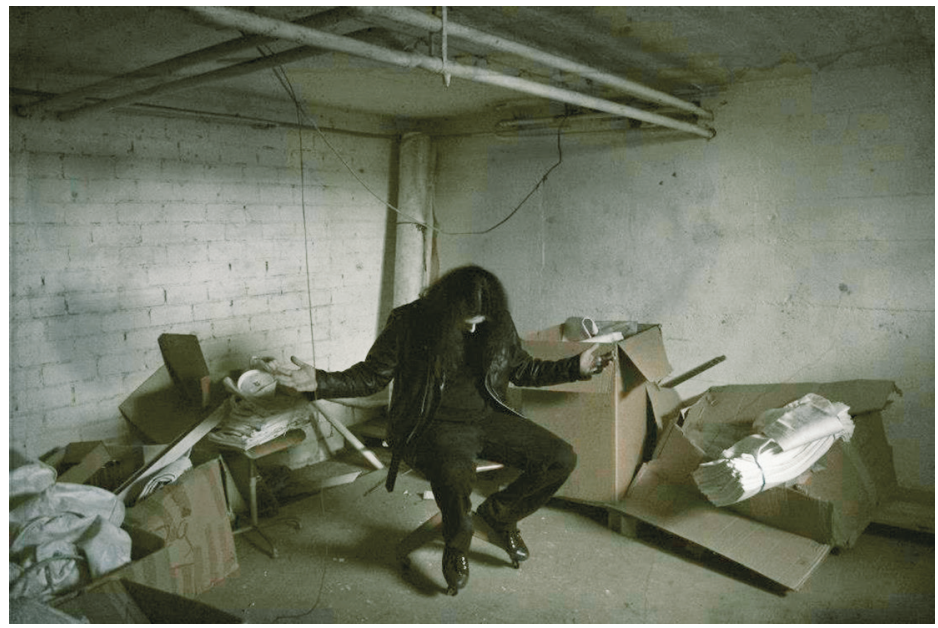

Abbildung 5: Ein Kellerabteil wird durch die Körperhaltung zum Gebetsraum. (C) Mille Hauser und Atritas 2012.

Bei spezifisch angelegten und bisweilen opulent ausgestatteten Gebetsräumen, in denen Menschen alleine oder im Kollektiv beten können, liegt der Fokus der Spatialität einerseits auf der öffentlicharchitektonischen Inszenierung und andererseits auf der Raumpraxis. Dabei spielt oft die Bewegung des Körpers eine Rolle: Das Schreiten im öffentlichen Raum als Teil der Gebetshandlung wird beispielsweise im Katholizismus in ganz unterschiedlicher Art und Weise gelebt: mimetische (Karfreitag) oder theophore (Fronleichnam) Prozessionen sind rituelle, feierliche Umzüge, die ein heilsgeschichtliches Geschehen nachzeichnen oder symbolisch Gott verehren, die aber auch der visuellen Inszenierung einer Glaubensgemeinschaft dienen. Durch Bittprozessionen werden dagegen Gottes Segen oder Schutz in einer konkreten Notsituation erbeten (Abb. 6). 


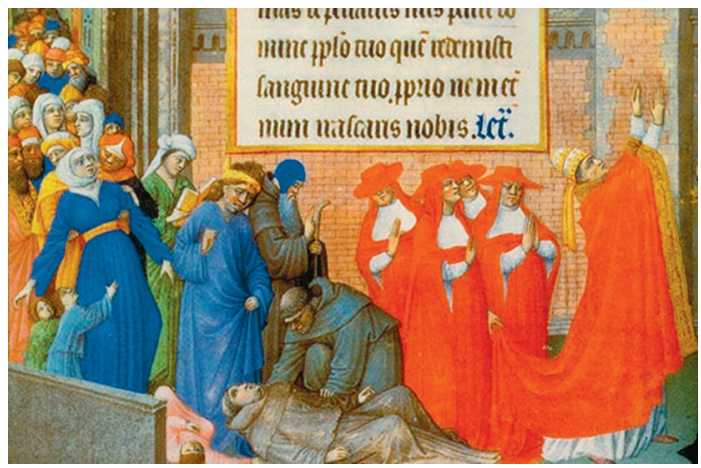

Abbildung 6: Miniatur in einem Gebetsbuch aus dem Beginn des 15. Jahrhunderts. Papst Gregor I. (590-604) leitet eine Prozession rund um Rom, um das Ende der Pest zu erflehen. Im Vordergrund zwei Opfer, ein Kind und ein Mönch:

http://commons.wikimedia.org/wiki/File:Plague01.jpg.

Auch im indischen Kontext wird eine Form des »bewegten Betens", nämlich das Umkreisen einer Götterstatue durch die Betenden bis heute praktiziert.

Ebenfalls auf ganz spezifische Art und Weise sind Körper, Gebet und Raum auf Pilgerreisen oder Wallfahrten, zum Beispiel im christlichen, islamischen oder New Age-Kontext miteinander verbunden: Die körperliche Anstrengung des Gehens oder Wanderns an einen - meist heiligen - Ort wird immer wieder durch Stationen des Gebets unterbrochen. Die individuelle Kommunikation mit einer transzendenten Macht während des Pilgerns ist selbstverständlich ebenfalls Teil des Prozesses. In der islamischen Tradition umkreisen die Wallfahrer am Ende ihrer Reise die Kaaba in Mekka und lobpreisen dabei Allah. Beten ist auch in dieser Hinsicht keine bloss sspirituell-geistige Handlung, sondern eine leibliche Kommunikation im und mit dem Raum.

Im Alltag kann ein religiöser Raum aber auch im kleineren Rahmen und temporär begrenzt geschaffen werden: Der muslimische Gebetsteppich und die Ausrichtung nach Mekka beim privaten Gebet formen beispielsweise visuell und materiell einen kleinen Raum, der abgehoben ist vom Alltag rundherum. Ist das Gebet beendet, wird auch dieser spezielle Raum "zusammengefaltet« und aufgelöst.

Das Kreieren eines "Gebetsraums" durch den Körper kann jedoch nicht nur im Innern eines Gebäudes oder im Freien stattfinden, sondern auch im Sinne einer wortwörtlichen Verinnerlichung, 
eines In-sich-Gehens. Im älteren Buddhismus wird beispielsweise kein Wesen ausserhalb angebetet, sondern das Gebet - falls man es so nennen möchte - richtet sich an den Buddha im Inneren. Der bekannte Lotussitz ist eine gängige Möglichkeit, sich entsprechend dieser Praxis zu vertiefen. Die wird z.B. in sogenannten esoterischen Gruppen in Mitteleuropa aufgenommen.

Das Zwiegespräch mit einer Transzendenz - ob ausserhalb oder innerhalb gedacht -, ist nicht an eine bestimmte, dafür reservierte Räumlichkeit gebunden, sondern kreiert anhand des Mediums Körper (Gestik, Bewegung, Regungslosigkeit) für eine spezifische Zeitspanne selbst einen religiösen Raum.

\section{Gebet in der Zeit}

"In the midnight hour I can feel your power Just like a prayer you know I'll take you there«

Madonna erfährt des Nachts die Macht oder die Präsenz Gottes beziehungsweise des schwarzen Heiligen. Weshalb es ausgerechnet Mitternacht sein musste, lässt im Rahmen dieses Songs Raum zur Spekulation:Vielleicht, weil dieser Zeitpunkt häufig als die Stunde zwischen Wachen und Träumen beschrieben wird, als Moment, in dem Nichterklärbares passieren kann, oder einfach, weil es gut klingt (Abb. 7a, b). Jedenfalls deutet die Sängerin an, dass Gebete, Zwiesprachen zwischen Mensch und Gottheit durchaus einen gewissen zeitlichen Aspekt aufweisen.

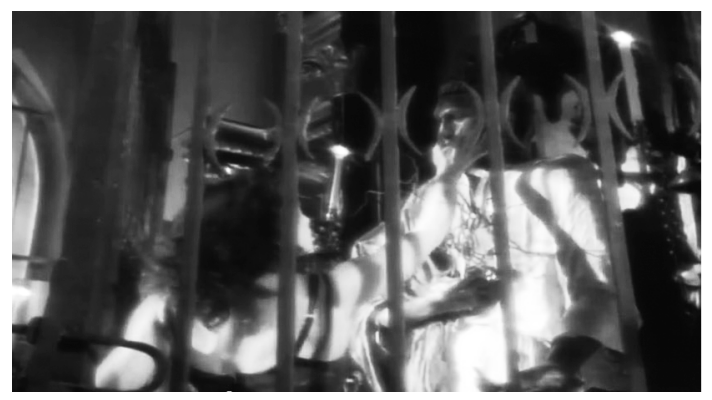

Abbildung $7 \mathrm{a}$ 


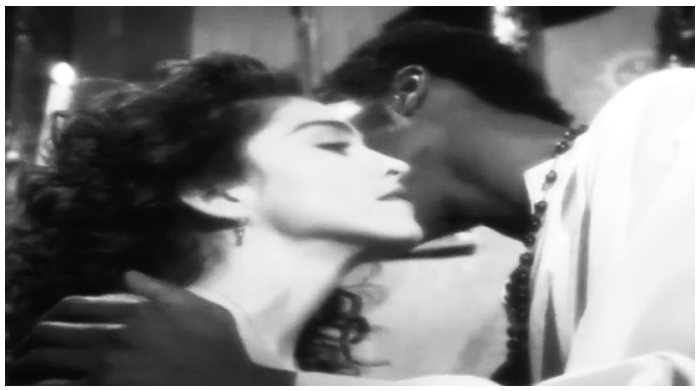

Abbildung 7a,b: Im Dämmerlicht reagiert der Heilige auf Madonnas inniges Gebet und weist ihr den richtigen Weg (Like a Prayer 00:01:45; 00:01:59).

Selbstverständlich kann - wie das Madonna-Beispiel zeigt - ein Stossgebet in einer Notsituation spontan improvisiert werden, dennoch ist Beten häufig Teil eines ritualisierten (Tages-)Ablaufs.

Wie bereits angedeutet, sind Gebetshandlungen häufig stark regulierte Abfolgen, die innerhalb eines Tages, einer spezifischen, mehrtägigen Zeitspanne (bspw. Karwoche) oder im Rahmen einer rituellen Zeremonie ihren ganz eigenen Platz haben. Gebete strukturieren den Tagesablauf und lösen den Körper, wie beispielsweise beim islamischen șalāt (dem rituellen Gebet, das fünf Mal täglich zu verrichten ist), für eine bestimmte Zeit aus seinem alltäglichen Rhythmus. Dieser Übergang von der einen in eine andere Phase wird oft durch die oben bereits beschrieben körperlichen Reinigungshandlungen eingeleitet.Viele religiöse Gemeinschaften kennen und praktizieren spezifische Reinigungsrituale vor dem eigentlichen Gebet (vgl. Abb. 8). Dabei sind die zu reinigenden Partien je nach religiöser $\mathrm{Zu}-$ gehörigkeit verschieden, häufig werden jedoch die für die Gestik relevanten Körperteile wie Gesicht oder Hände gesäubert.

In gewissen Traditionen wird der Körper vor dem eigentlichen Akt des Betens in ein spezifisches Kleidungsstück gehüllt. Der Tallit, ein jüdischer Gebetsmantel, wird beispielsweise seit der Neuzeit von erwachsenen Juden (und im liberalen Judentum auch von Jüdinnen) beim Morgengebet getragen. Das den Kopf und die Schultern verhüllende Tuch wird in Kombination mit der Körperhaltung zum untrüglichen Zeichen einer religiösen Praxis (Abb. 9). 


\section{DIE TEILWASCHUNG "WUDU"}
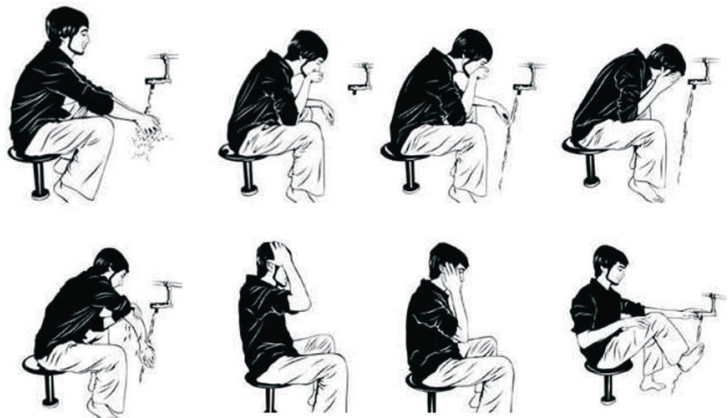

Abbildung 8: Anleitung zur Waschung vor dem Gebet für Muslime:

http://islamimherzen.de/die-teilwaschung-wudu-ganzkoerperwaschung-ghusl/

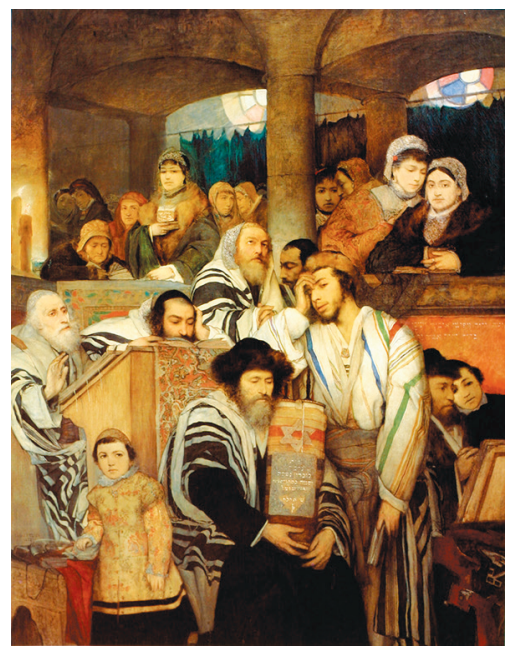

Abbildung 9: Maurycy Gottlieb, Jews Praying in the Synagogue on Yom Kippur, 1878.

Öl auf Leinwand, 245x192 cm, Tel Aviv Museum of Art 
Das Anziehen der spezifischen Kleidung signalisiert zusätzlich den Übergang vom Alltag zum rituellen Anrufen oder Lobpreis der transzendenten Macht. Sind diese vorbereitenden Handlungen durchgeführt, kann die eigentliche Gebetsphase beginnen.

Der Rosenkranz oder ähnliche Gebetsketten, wie sie sich im Islam (misbaha), dem Hinduismus oder Buddhismus (mala) finden, unterstützen auf sehr körperliche Weise die zeitliche Wahrnehmung und die Struktur des Ablaufs hinsichtlich eines sich wiederholenden Gebetes. Die Materialität der Gebetskette stellt in diesem Sinne die Verbindung zwischen Alltag und Auszeit dar, die dem im Gebet versunkenen Körper anzeigt, an welchem konkreten Punkt des Kommunikationsprozesses er sich befindet. Das Gebet selbst wird zu einer Art Auszeit vom Alltag, da äusserliche Faktoren zumindest kurzzeitig ausgeblendet werden. Der Abschluss eines Gebets und die Überleitung ins Alltägliche werden dann wieder durch bestimmte Gesten wie beispielsweise das Kreuzzeichen im römisch-katholischen Kontext signalisiert.

\section{Dem Alltag enthobene Alltagshandlung}

"Just like a muse to me, you are a mystery

Just like a dream, you are not what you seem

Just like a prayer, no choice your voice can take me there

Die Auseinandersetzung mit einer transzendenten Macht, das führt nicht nur Madonna vor Augen, ist eine Art der Kommunikation, die stets mit einem gewissen Mass an Unsicherheit, an Rätselhaftigkeit verbunden ist. Regulierungen bezüglich Gebetshaltung, Texten, Lautstärke oder auch Bekleidung können dementsprechend hilfreich sein. Auch die Praxis des gemeinsamen Betens oder des Gebets zu einer spezifischen Zeit an einem bestimmten Ort kann den Aspekt des Unerklärlichen in eine alltägliche Struktur einordnen und Orientierung generieren. Dadurch, dass diese Art der Kommunikation hauptsächlich körperbasiert ist, wird einerseits die Verhaftung in der Welt und andererseits die performative Qualität des Gebets allein oder innerhalb der Gruppe deutlich.

Wenden wir uns noch einmal dem Beispiel des >Fussball-Gebets zu: Die Fussballspieler, die sich vor dem Match im Kreis formieren um zu beten, und die Fans, die während der Verlängerung Stossgebete gen Himmel schicken, sie alle benutzen ihren Körper als Medium der Kommunikation mit der Gottheit. Die Inszenierung 
im Betkreis wirkt etwas einstudiert, wohingegen sich die plötzlichen, emotionalen Ausbrüche der Zuschauer und Zuschauerinnen in individuellen Gebetshaltungen offenbaren. Diese sind im öffentlichen, rituellen Kontext wesentlich strukturierter, was aber nicht heissen soll, dass die individuelle Kommunikation mit einer Gottheit nicht auch bestimmten körperlichen Kriterien folgen kann, wie es etwa bei Tagesgebeten der Fall ist. Die Inszenierung des betenden Körpers im Raum widerspiegelt sowohl die persönliche Frömmigkeit als auch die Selbstwahrnehmung einer Gemeinschaft. Die betende brasilianische Nationalmannschaft im Stadion verweist somit auf den zumeist christlichen Hintergrund ihrer einzelnen Spieler und steht sinnbildlich für das einige Brasilien, genauso wie die Fans ihre persönliche religiöse Ausrichtung dazu nutzen, sich Hilfe zu erbitten.

Eine Zwiesprache mit einer transzendenten Macht unterscheidet sich in wesentlichen Punkten vom alltäglichen Gespräch zwischen zwei menschlichen Individuen. Zwar gehört für viele das Beten zum Alltag, strukturiert gar den Tagesablauf, aber dennoch ist der spezifische Akt der Kontaktaufnahme mit einer Gottheit ein aussergewöhnlicher und dem Alltag enthobener Moment - eine Paradoxie, die dem Gebet anhaftet und nicht gelöst werden sollte: Der ganze Körper, jede einzelne Faser ist angespannt oder total entspannt; alle Sinne sind geschärft um in einen Dialog zu treten. Der Körper wird Mittel zum Zweck, temporär jeder räumlichen und zeitlichen Ordnung enthoben. Oder wie Madonna singt: »It's like a dream - No end and no beginning - You're here with me it's like a dream - Let the choir sing."

— Natalie Fritz ist Doktorandin der Religionswissenschaft am Zentrum für Religion, Wirtschaft und Politik an der Theologischen Fakultät der Universität Zürich.

- Dr. Anna-Katharina Höpflinger ist Oberassistentin am Zentrum für Religion, Wirtschaft und Politik an der Theologischen Fakultät der Universität Zürich. 